\title{
ON THE RELATION BETWEEN POSITIVE DEFINITE FUNCTIONS AND GENERALIZED TOEPLITZ KERNELS
}

\author{
J. FRIEDRICH
}

(Communicated by Palle E. T. Jorgensen)

\begin{abstract}
We show that extension problems for generalized Toeplitz kernels may be completely reduced to extension problems for positive definite functions, where the solution is well known. These considerations in particular imply that generalized Toeplitz kernels may be represented as Fourier transforms of positive operator-valued measures.
\end{abstract}

The notion of generalized Toeplitz kernels (g.T.k.) was introduced in [5], where also a generalized Bochner theorem was proved in the discrete case. Since then many papers concerning this topic appeared, for example, $[2,3]$. The extension problem for the discrete case was discussed in [1] and for the continuous case, for example, in [4]. The proofs for the extendibility and for the generalized Bochner theorem use mainly lifting theorems for families of operators. In this paper we want to show that these questions can be reduced directly to appropriate questions for positive definite operator-valued functions.

Fix Hilbert spaces $H_{1}, H_{2}$, and let $0<a \leq \infty, I(a):=(-2 a, 2 a), I_{1}(a):=$ $(-2 a, 0), I_{2}(a):=(0,2 a)$. An operator-valued generalized Toeplitz kernel $K$ on $I(a) \times I(a)$ is an operator-valued function with $K(s, t) \in L\left(H_{\alpha}, H_{\beta}\right)$ if $s \in I_{\alpha}(a), t \in I_{\beta}(a)$ and if there are four weakly continuous functions

$$
K_{\beta \alpha}: I_{\alpha}(a)-I_{\beta}(a) \rightarrow L\left(H_{\alpha}, H_{\beta}\right), \quad \alpha, \beta=1,2,
$$

such that $K(s, t)=K_{\beta \alpha}(s-t)$ for $s \in I_{\alpha}(a), t \in I_{\beta}(a)$, and $K_{12}(-t)=K_{21}(t)^{*}$ for $t \in(0,4 a)$; i.e., $K_{11}:(-2 a, 2 a) \rightarrow L\left(H_{1}\right), K_{12}:(-4 a, 0) \rightarrow L\left(H_{2}, H_{1}\right)$, $K_{21}:(0,4 a) \rightarrow L\left(H_{1}, H_{2}\right), K_{22}:(-2 a, 2 a) \rightarrow L\left(H_{2}\right)$.

A g.T.k. is called positive definite if

$$
\sum_{\alpha, \beta=1,2} \sum_{s \in I_{\alpha}(a), t \in I_{\beta}(a)}\left\langle K_{\beta \alpha}(s-t) \varphi_{\alpha}(s), \varphi_{\beta}(t)\right\rangle_{H_{\beta}} \geq 0
$$

for all pairs of functions $\varphi_{\alpha}: I\left(\alpha(a) \rightarrow H_{\alpha}, \alpha=1,2\right.$, with finite support.

Recall that an operator-valued function $F:(-2 a, 2 a) \rightarrow L(H)$ with some Hilbert space $H$ is called positive definite if

$$
\sum_{s, t \in(-a, a)}\langle F(s-t) \phi(s), \phi(t)\rangle_{H} \geq 0
$$

Received by the editors May 29, 1992.

1991 Mathematics Subject Classification. Primary 43A35, 42A82, 47A20; Secondary 46L30. 
for all functions $\phi:(-a, a) \rightarrow H$ with finite support.

Suppose we are given a g.T.k. $K$ on $I(a) \times I(a), 0<a<\infty$. We associate a function $F_{K}:(-2 a, 2 a) \rightarrow H, H:=H_{1} \oplus H_{2}$, to $K$ defining

$$
F_{K}(t)=\left(\begin{array}{cc}
K_{11}(t) & K_{12}(t-2 a) \\
K_{21}(t+2 a) & K_{22}(t)
\end{array}\right), \quad t \in(-2 a, 2 a) .
$$

Here and in the following we use the canonical matrix representation of bounded operators w.r.t. the decomposition $H=H_{1} \oplus H_{2}$.

Proposition 1. The generalized Toeplitz kernel $K$ is positive definite if and only if $F_{K}$ is positive definite.

Proof. The proof follows from a straightforward calculation, if we relate the functions appearing in the definitions by

$$
\phi(t)=\left(\begin{array}{l}
\varphi_{1}(t+a) \\
\varphi_{2}(t-a)
\end{array}\right), \quad t \in(-a, a) .
$$

Since a g.T.k. $K$ is weakly continuous by definition, $F_{K}$ is weakly continuous, too. For weakly continuous operator-valued positive definite functions $F$ on $(-2 a, 2 a)$ there is always a positive definite extension $\widetilde{F}$ to the real line (cf. [7]). As it was stated, for example, [6, Lemma 2], such functions are automatically uniformly strongly continuous. More exactly,

$$
\begin{aligned}
& \|(\tilde{F}(t)-\tilde{F}(t+h)) \phi\|^{4} \\
& \quad \leq 16\|\widetilde{F}\|^{3}|\langle(\tilde{F}(0)-\tilde{F}(h)) \phi, \phi\rangle|, \quad \phi \in H, t, h \in \mathbb{R} .
\end{aligned}
$$

If $|h|<2 a$, we are allowed to omit the tildes on the right-hand side, since $\widetilde{F}$ coincides with $F$ on $(-2 a, 2 a)$. In particular, the set of all p.d. extensions $\widetilde{F}$ of $F$ is strongly equicontinuous.

Suppose now that $\widetilde{F}$ is a positive definite extension of $F_{K}$, where $K$ is a positive definite g.T.k. Then we define an operator-valued function $\widetilde{K}$ by

$$
\tilde{K}(t)=\left(\begin{array}{cc}
\widetilde{F}_{11}(t) & \widetilde{F}_{12}(t+2 a) \\
\widetilde{F}_{21}(t-2 a) & \widetilde{F}_{22}(t)
\end{array}\right), \quad t \in \mathbb{R} .
$$

Let $\widetilde{K}_{\alpha \beta}$ denote the entries of the corresponding matrix representation of $\widetilde{K}$. Then $\widetilde{K}_{\alpha \beta}(t)=K_{\alpha \beta}(t), \alpha, \beta=1,2$, if the right-hand side is defined. Moreover, $\widetilde{K}$ is an operator-valued positive definite function in the usual sense. Its positive definiteness follows immediately from that one of $\widetilde{F}$.

A positive definite function $\widetilde{K}$, whose entries in the canonical matrix representation coincide with the constituents of $K$, where the latter are defined, will be called a positive definite extension of $K$.

The strongly continuous positive definite functions on $\mathbb{R}$ with values in $L(H)$ form a subset of a linear space $E$ of all strongly continuous functions on $\mathbb{R}$ with values in $L(H)$. On $E$ we introduce a locally convex topology by the seminorms

$$
E \ni F \rightarrow\left|\langle F(t) \phi, \psi\rangle_{H}\right|, \quad t \in \mathbb{R}, \phi, \psi \in H .
$$

The corresponding induced topology is meant in the following if topological questions are discussed for sets of strongly continuous positive definite functions. 
Lemma 2. Suppose that $K$ is a positive definite g.T.k. on $I(a) \times I(a), 0<a<$ $\infty$. Then the set $\mathscr{M}_{\alpha}$ of all positive definite extensions of $K$ is nonempty and compact.

Proof. The assertion $\mathscr{M}_{a} \neq \varnothing$ was just shown.

To prove compactness, we consider an ultrafilter $\mathscr{U}$ on $\mathscr{M}_{a}$. Since

$$
|\langle\widetilde{K}(t) \phi, \psi\rangle| \leq\|\widetilde{K}(0)\|\|\phi\|\|\psi\|
$$

and since the operator $\widetilde{K}(0)$ is the same for all $\widetilde{K} \in \mathscr{M}_{a}$ because of weak continuity, the limit $g(t ; \phi, \psi)=\lim _{\mathscr{U}}\langle\widetilde{K}(t) \phi, \psi\rangle$ exists. Moreover, $g(t ; \phi, \psi)$ is linear w.r.t. $\phi$ and antilinear w.r.t. $\psi$. Thus it defines a continuous sesquilinear form on $H$, i.e., there is a unique $G(t) \in L(H)$ such that

$$
g(t ; \phi, \psi)=\langle G(t) \phi, \psi\rangle, \quad \phi, \psi \in H, t \in \mathbb{R} .
$$

The operator-valued function $t \rightarrow G(t)$ is positive definite. To see this, we simply have to fix a function $\phi: \mathbb{R} \rightarrow H$ with finite support and to perform the limit in (1). It remains to show that $G$ is weakly continuous. Since the function $G^{\prime}$ defined by

$$
G^{\prime}(t)=\left(\begin{array}{cc}
G_{11}(t) & G_{12}(t-2 a) \\
G_{21}(t+2 a) & G_{22}(t)
\end{array}\right), \quad t \in \mathbb{R}
$$

is positive definite and coincides with $F_{K}$ on $(-2 a, 2 a)$, its weak continuity follows from that one of $F_{K}$, resp. $K$. Now it is easy to see that the weak continuity of $G^{\prime}$ implies the weak continuity of $G$.

Since all $\widetilde{K} \in \mathscr{M}_{a}$ are extensions of $K$, i.e., the entries of the appropriate matrix functions are equal to the constituencies of $K$ where the latter are defined, the same is true for $G$, i.e., $G \in \mathscr{M}_{a}$. Since $g(t ; \phi, \psi)=\langle G(t) \phi, \psi\rangle$ in (3), we obtain finally $G=\lim _{\mathscr{U}} \widetilde{K}$, which completes the proof.

Theorem 1. Suppose that $K$ is a positive definite g.T.k. on $I(a) \times I(a), 0<a \leq$ $\infty$. Then the set $\mathscr{M}_{a}$ of all positive definite extensions of $K$ is nonempty and compact.

Proof. For $a<\infty$ the assertion is Lemma 2. For $a=\infty$, we denote by $\mathscr{M}_{b}, 0<b<\infty$, the set of all positive definite extensions of the restriction of $K$ to $I(b) \times I(b)$. Then $\mathscr{M}_{\infty}=\bigcap_{0<b<\infty} \mathscr{M}_{b}$. Since all sets $\mathscr{M}_{b}$ are compact and nonempty, and, since $\mathscr{M}_{b} \subset \mathscr{M}_{b^{\prime}}$ if $b^{\prime}<b$, their intersection is nonempty and compact.

Corollary 3. Any positive definite g.T.k. may be represented as the Fourier transform of a positive operator-valued measure.

Proof. This follows immediately from our theorem and the fact that any weakly continuous positive definite operator-valued function is the Fourier transform of a positive measure.

Remark. Similar considerations as above apply to the discrete case, i.e., if $(-2 a, 2 a)$ is replaced by $\{-2 a,-2 a+1, \ldots, 2 a-1,2 a\}$, where $2 a$ is a positive integer. 


\section{REFERENCES}

1. R. Arocena, On the extension problem for a class of translation invariant positive forms, J. Operator Theory 21 (1989), 323-347.

2. R. Arocena and M. Cotlar, Dilation of generalized Toeplitz kernels and some vectorial moment and weighted problems, Lecture Notes in Math., vol. 908, Springer, Berlin, 1982.

3. __ Generalized Toeplitz kernels and Adamjan-Arov-Krein moment problems, Oper. Theory: Adv. Appl., vol. 4, Birkhauser, Basel and Boston, MA, 1982, pp. 37-55.

4. R. Bruzual, Local semigroups of contractions and some applications to Fourier representation theorems, Integral Equations Operator Theory 10 (1987), 780-801.

5. M. Cotlar and C. Sadosky, On the Helson-Szegö theorem and a related class of modified Toeplitz kernels, Amer. Math. Soc., Providence, RI, 1979, pp. 383-407.

6. J. Friedrich, Integral representations of positive definite matrix-valued distributions on cylinders, Trans. Amer. Math. Soc. 313 (1989), 275-299.

7. M. L. Gorbachuk, On representations of positive definite operator functions, Ukrain. Mat. Z. 17 (1965), 29-45. (Russian)

F. B. MAthemetik, UNiversität Leipzig, Augustusplatz 10/11, 0-7010 Leipzig, GermaNy Current address: Rosa-Luxemburg Strasse 21, 04509 Delitzsch, Germany 